\title{
CARACTERÍSTICAS FÍSICO-QUÍMICAS E ACEITAÇÃO SENSORIAL DE APRESUNTADO COM ADIÇÃO DE FIBRA DE ERVILHA VISANDO REDUÇÃO DE CUSTO
}

\section{PHYSICOCHEMICAL PARAMETERS AND SENSORY ACCEPTANCE OF CHOPPED HAM WITH PEA FIBER AIMING AT COST REDUCTION}

\author{
Yana Jorge Polizer ${ }^{*}$ \\ Daniel Pompeu² \\ Raul Pereira Fregonesi ${ }^{1}$ \\ Merícia Harumi Hirano ${ }^{1}$ \\ Maria Teresa de Alvarenga Freire ${ }^{1}$ \\ Marco Antonio Trindade ${ }^{1}$

\begin{abstract}
${ }^{1}$ Faculdade de Zootecnia e Engenharia de Alimentos da Universidade de São Paulo, Pirassununga, SP, Brasil. 2Labonathus Biotecnologia Internacional, São Paulo, SP, Brasil.

*Autora para correspondência - yana.polizer@terra.com.br
\end{abstract}

\section{Resumo}

O objetivo deste trabalho foi desenvolver e avaliar as características físico-químicas e sensoriais de apresuntado adicionado de fibra de ervilha como substituto parcial de carne visando redução de custos. Foram processadas duas formulações: Controle - semelhante à formulação comercial e Fibra/Menos Carne (FMC) - redução de 5\% de carne e adição de 1\% de fibra de ervilha mais $4 \%$ de água. Após o processamento, os produtos foram caracterizados quanto a $\mathrm{pH}$, perfil de textura, cor objetiva, perdas por gotejamento, composição centesimal e avaliação sensorial (teste de aceitação com escala hedônica de nove pontos). $\mathrm{Na}$ análise do perfil de textura, os parâmetros dureza e mastigabilidade foram maiores $(\mathrm{p}<0,05)$ na formulação Controle. A formulação FMC apresentou valor de $\mathrm{L}^{*}$ superior $(\mathrm{p}<0,05)$ e valores de $\mathrm{a}^{*}$ e $\mathrm{b}^{*}$ inferiores $(\mathrm{p}<0,05)$ ao tratamento Controle. Quanto à avaliação sensorial, os atributos aroma, aparência, textura e sabor não apresentaram diferenças $(p>0,05)$. Já em relação à aceitação geral as formulações diferiram $(p<0,05)$, sendo que FMC apresentou maior aceitabilidade em comparação ao Controle. Conclui-se que é possível utilizar a fibra de ervilha em apresuntado substituindo parcialmente a carne, gerando um produto com boa aceitação sensorial e menores custos.

Palavras-chave: aceitação sensorial; fibras alimentares; produtos cárneos; redução de custo; textura.

\begin{abstract}
This study was developed to evaluate the physicochemical and sensorial characteristics of chopped ham prepared with the addition of pea fiber as a partial substitute of meat. Two treatment samples were processed: Control (C)-commercial production; and Fiber Less Meat (FLM)-reduction of 5\% of meat and addition of $1 \%$ of fiber and $4 \%$ of water. After processing, the products were characterized regarding $\mathrm{pH}$ levels, texture, objective color, loss by dripping, centesimal composition, scanning electron microscopy (SEM), and sensorial evaluation (9-point hedonic scale acceptability test). Analyzing the texture, the parameters for firmness and chewy were higher $(\mathrm{p}<0.05)$ for the $\mathrm{C}$
\end{abstract}


sample. The sample FLM presented higher $\mathrm{L}^{*}$ value $(\mathrm{p}<0.05)$ and lower $\mathrm{a}^{*}$ and $\mathrm{b}^{*}$ values $(\mathrm{p}<0.05)$ than sample C. Regarding the sensorial evaluation, the items aroma, appearance, texture, and flavor did not present differences ( $p>0.05)$. Regarding general acceptability, the samples differed $(p<0.05)$ and the FLM sample presented higher acceptability compared to the control sample. In conclusion, it is possible to use pea fibers in chopped ham as a partial substitute for meat having a final product with high sensorial acceptability and cost reduction.

Keywords: cost reduction; food fibers; meat products; sensorial acceptability; texture.

Recebido em: 16 de julho de 2014

Aceito em: 13 de dezembro de 2017

\section{Introdução}

A carne suína é a forma de proteína animal mais consumida no mundo. Isso se deve aos vários aspectos que facilitam sua transformação, além de oferecer inúmeras opções de venda no mercado ${ }^{(1)}$. Enquanto a carne bovina é mais consumida in natura, a carne suína tem $70 \%$ do seu consumo feito na forma de embutidos ${ }^{(2)}$. A industrialização torna-se uma alternativa para o escoamento dessa matéria-prima, além de proporcionar um aumento na vida útil de produtos, como no caso dos processados cárneos.

A adição de fibras dietéticas em produtos cárneos é excelente na substituição parcial do conteúdo cárneo desses produtos por seus inerentes efeitos funcionais e nutricionais, mantendo a qualidade sensorial e reduzindo o custo de produção desses produtos ${ }^{(3-6)}$. Além de benefícios à saúde, a incorporação de fibras dietéticas em produtos cárneos aumenta o volume, reduz perdas no cozimento e não provoca nenhuma ou poucas alterações nos parâmetros de textura, por melhorar a capacidade de retenção de água, trazendo, dessa forma, grandes vantagens tanto para consumidores quanto para os processadores $^{(3)}$.

As principais fibras usadas em produtos cárneos cozidos são de laranja, beterraba, trigo, aveia e ervilha $^{(7)}$. O produto comercial conhecido como fibra de ervilha pode ser definido como uma mistura extraída da ervilha, composta essencialmente por fibras, amido e proteínas (Roquette Freres, France). $\mathrm{O}$ uso dessa fibra em produtos cárneos tem sido investigado pelas características e propriedades apresentadas (facilidade de dispersão em água e óleo, capacidade de absorção de líquidos, fácil liga à fibra de temperos e essências, paladar neutro e livre de odor).

O objetivo deste trabalho foi avaliar as características físico-químicas e sensoriais de apresuntado elaborado com adição de fibra de ervilha como substituto parcial da carne suína.

\section{Material e Métodos}

No delineamento experimental foram desenvolvidas duas formulações para o apresuntado (Tabela 1): 1) Controle (C): sem fibra de ervilha, semelhante a uma formulação comercial e 2) Fibra/Menos Carne (FMC): redução de 5\% de carne suína, adição de 1\% de fibra de ervilha e $4 \%$ de água. Todo o experimento foi repetido duas vezes. 
Tabela 1. Formulações utilizadas na elaboração dos apresuntados

\begin{tabular}{lcc}
\hline & Controle & Fibra/Menos Carne \\
\hline Ingredientes & $\mathbf{\%}(\mathbf{m} / \mathbf{m})$ & $\mathbf{\%}(\mathbf{m} / \mathbf{m})$ \\
\hline Paleta Suína & 80 & 75 \\
Fibra de Ervilha & - & 1 \\
Água & 16 & 20 \\
Mix para Apresuntado & 4 & 4 \\
\hline
\end{tabular}

Para o processamento do apresuntado, a paleta suína utilizada no presente trabalho foi adquirida no comércio local de Pirassununga - SP e passou por uma toalete para remoções de tecido conjuntivo aparente e cobertura de gordura. A fibra de ervilha (Pea Fiber I50M® - Roquette Freres, France) utilizada foi fornecida pela empresa Labonathus Biotecnologia Internacional Ltda.

De acordo com o fabricante (Roquette Freres, France), a fibra de ervilha utilizada no presente estudo apresenta a seguinte composição: teor de umidade de 10\% (máximo), teor de fibra (base seca) de 50\% máximo, teor de proteínas (base seca) de 10\% (máximo) e amido (base seca) de aproximadamente 35\%. O mix para apresuntado Doremax (Doremus Alimentos Ltda) continha todos os condimentos e aditivos necessários (sal, condimentos, nitrito de sódio, antioxidante eritorbato de sódio e estabilizante tripolifosfato de sódio).

As matérias-primas foram pesadas, separadamente, assim como todos os ingredientes e a água. A carne suína foi moída em moedor elétrico, com disco de $16 \mathrm{~mm}$. Em seguida, a carne e os demais ingredientes foram misturados por dez minutos até a obtenção de massa homogênea. A massa resultante foi embutida com o auxílio de uma embutidora pneumática (V25, Sirman) em tripa de poliamida e enformada em forma metálica com peças de aproximadamente $1 \mathrm{~kg}$. Após esse período, os apresuntados foram imersos em água aquecida a $70^{\circ} \mathrm{C}$ por uma hora e em seguida a $80^{\circ} \mathrm{C}$ até o as peças atingirem temperatura interna de $72{ }^{\circ} \mathrm{C}$. Após o cozimento, foi realizado resfriamento por imersão em água corrente, até as peças atingirem temperatura interna de $45^{\circ} \mathrm{C}$. Posteriormente, as peças foram acondicionadas sob refrigeração por 24 horas antes de serem desenformadas. Após esse período, os produtos foram desenformados e acondicionados sob refrigeração $\left(4^{\circ} \mathrm{C}\right)$ até o momento das análises.

A composição centesimal foi determinada por meio da metodologia oficial da Association of Official Analytical Chemistry ${ }^{(8)}$. Dessa maneira foram determinadas umidade (950.46), resíduo mineral fixo ou cinzas (920.153) e proteínas (928.08). O teor de lipídeos foi determinado pelo método de Bligh e Dyer $^{(9)}$.

A determinação de $\mathrm{pH}$ foi realizada através de pHmetro portátil (Modelo HI 99163, Marca HANNA) com eletrodo de perfuração em três pontos de cada amostra.

As amostras foram submetidas a análise de cor com um colorímetro portátil (Modelo MiniScan XE, Marca HunterLab), empregando escala $L^{*}$, $a^{*}$ e $b^{*}$ do sistema de avaliação CIELab - "Comissão Internationate de L'Eclairage", utilizando-se o iluminante D65, ângulo de observação de $10^{\circ} \mathrm{e}$ abertura de célula com $30 \mathrm{~mm}$ para cálculo dos valores $\mathrm{L}^{*}$ (luminosidade), a* (intensidade de cor verde-vermelho) e $b^{*}$ (intensidade de cor azul-amarelo). Foram tomadas três medidas em diferentes pontos de cada amostra. 
Para a análise de perda de água por gotejamento, as amostras foram cortadas em cubos de $3,5 \mathrm{~cm}$ que foram pesados em balança semianalítica para se obter o peso inicial (Pi). Esses cubos foram envolvidos por uma embalagem plástica e mantidos suspensos em refrigerador doméstico à temperatura de aproximadamente $4{ }^{\circ} \mathrm{C}$. Depois de 24 horas, as amostras foram secas com papel toalha e pesadas, obtendo-se o peso final (Pf). Os resultados foram calculados pela equação (1):

$$
P G=\frac{M_{i}-M_{f}}{M_{i}} \cdot 100
$$

A análise do perfil de textura instrumental (TPA) foi realizada em texturômetro (TAXT2i, Stable Micro Systems). As amostras foram previamente cortadas em cubos de $2 \mathrm{~cm}$, comprimidas duas vezes até 50\% de seu tamanho e não houve tempo de repouso da amostra entre os dois ciclos de compressão. A curva de deformação com o tempo foi obtida a uma velocidade de compressão de $3 \mathrm{~mm} / \mathrm{s}$ e os parâmetros estudados foram: dureza (g), elasticidade (adimensional) e mastigabilidade (g.mm). A análise foi realizada em dez amostras para cada tratamento.

Para a avaliação sensorial, as amostras foram cortadas em fatias de aproximadamente $2 \mathrm{~mm}$ e servidas à temperatura de aproximadamente $10^{\circ} \mathrm{C}$ (temperatura na qual o produto é normalmente consumido), em pratos codificados com número de três dígitos casualizados e analisados em cabines individuais.

Foi realizado um teste afetivo de aceitação com 60 consumidores habituais de presunto/apresuntado utilizando escala hedônica estruturada de nove pontos, variando de "gostei extremamente", com nota igual a 9, a "desgostei extremamente", com nota igual a 1. Os atributos avaliados foram aroma, aparência, textura, sabor e aceitabilidade geral. Os consumidores receberam um Termo de Consentimento Livre e Esclarecido para ser lido e assinado antes da realização da avaliação sensorial. O presente trabalho foi aprovado pelo Comitê de Ética da Faculdade de Zootecnia e Engenharia de Alimentos da USP, de acordo com o número do parecer: 122.191 (CAAE: 08408612.6.0000.5422).

Os resultados das análises foram submetidos à análise de variância (ANOVA) e teste de Tukey para comparação das médias. Utilizou-se o programa SAS (Statistic Analisis System), considerando 5\% de nível de significância.

\section{Resultados e Discussão}

Os resultados da composição centesimal estão expressos na Tabela 2. A formulação FMC apresentou valor superior $(p<0,05)$ em relação ao teor de umidade e cinzas. $O$ aumento no teor de umidade no tratamento FMC foi proporcional à quantidade de água adicionada nesse tratamento (4\%) em relação ao C. No presente trabalho, somente a formulação $\mathrm{C}$ atendeu ao valor de umidade estabelecido em legislação brasileira para apresuntado ${ }^{(10)}$, que estabelece valores máximos de $75 \%$. Bessera et al. ${ }^{(11)}$ encontraram valores de umidade em apresuntado de carne suína e caprina (acrescidos de $2 \%$ de fécula de mandioca) de $74,19 \%$, valor este muito próximo ao encontrado no presente estudo. Resultados similares também foram relatados por Pedroso et al. ${ }^{(12)}$ que, ao avaliarem a adição de amido ( 0 a $\left.2 \%\right)$ e carragena ( 0 a 1\%) em presunto cozido de peru, encontraram teores de umidade entre 74,06 a $75,85 \%$.

Foram encontrados maiores $(\mathrm{p}<0,05)$ teores de proteína e lipídeos para a formulação $\mathrm{C}$. Tal resultado é decorrente da redução de $5 \%$ do conteúdo cárneo (principal fonte proteica/gordura intramuscular) 
na formulação FMC. Sordi et al. ${ }^{(13)}$, que estudaram presunto cozido acrescido de $1,5 \%$ de fibra solúvel de trigo, encontraram composição centesimal de: 70,11\% de umidade, 20,40\% de proteína, 1,38\% de lipídeos e $4 \%$ de cinzas, valores estes ligeiramente diferentes aos encontrado no presente trabalho. As duas formulações testadas no presente estudo atenderam aos teores de proteína e gordura estabelecidos nos padrões da legislação brasileira $^{(10)}$, apresentando valores acima do mínimo exigido para o conteúdo proteico $(13,0 \%)$ e abaixo do máximo exigido para o teor de gordura $(12 \%)$.

Tabela 2. Composição centesimal (média \pm erro padrão) das formulações de apresuntado

\begin{tabular}{ccc}
\hline & $\mathbf{C}^{\mathbf{l}}$ & FMC $^{\mathbf{2}}$ \\
\hline Umidade (\%) & $74,81 \pm 0,03^{\mathrm{b}}$ & $77,15 \pm 0,005^{\mathrm{a}}$ \\
Cinzas (\%) & $3,61 \pm 0,01^{\mathrm{b}}$ & $3,91 \pm 0,005^{\mathrm{a}}$ \\
Lipídeos (\%) & $2,75 \pm 0,03^{\mathrm{a}}$ & $2,10 \pm 0,005^{\mathrm{b}}$ \\
Proteínas (\%) & $19,78 \pm 0,08^{\mathrm{a}}$ & $17,32 \pm 0,005^{\mathrm{b}}$ \\
\hline
\end{tabular}

${ }^{1}$ Controle: formulação similar a comercial; ${ }^{2} \mathrm{Fibra} / \mathrm{Menos}$ Carne: redução de $5 \%$ de carne, adição de $1 \%$ de fibra de ervilha e $4 \%$ de água.

Letras minúsculas diferentes em uma mesma linha indicam diferenças significativas $(p<0.05)$ entre os tratamentos.

Adição de fibra de ervilha (1\%) e a redução de carne $(5 \%)$ não afetou o $\mathrm{pH}(\mathrm{p}>0,05)$ do produto (Tabela 3). Os resultados observados de $\mathrm{pH}$ foram similares aos encontrados por Pedroso et al. ${ }^{(12)}$ que, ao avaliarem a adição de amido de mandioca ( 0 a $2 \%$ ) e carragena ( 0 a $1 \%)$ em presunto cozido de peru, também não encontraram diferenças em relação a estes valores. Resultados diferentes foram reportados por Huber ${ }^{(14)}$ que, ao avaliar os valores de $\mathrm{pH}$ de produtos cárneos reestruturados de frango (hambúrguer e empanado) com adição de mix de fibras vegetais de bambu, trigo e ervilha (0,4 a $1,6 \%$ ) como substitutos totais de gordura, relataram que os valores de $\mathrm{pH}$ apresentaram diferenças entre os tratamentos $(\mathrm{p}<0,05)$ em estudo. Junior et al. ${ }^{(15)}$ também encontraram diferenças nos valores de $\mathrm{pH}$ de hambúrguer adicionado de farinha de aveia (0 a 4\%), os valores de $\mathrm{pH}$ das formulações variaram entre 4,32 a 5,17. As diferenças encontradas na literatura com relação ao valor de $\mathrm{pH}$ podem estar relacionadas à quantidade de fibra empregada nas formulações. Como a adição de fibra de ervilha no presente trabalho foi realizada em baixo percentual (1\%), possivelmente o $\mathrm{pH}$ da mesma exerceu pouca influência sobre o $\mathrm{pH}$ do produto final.

Com relação às variáveis de cor, a formulação $\mathrm{FMC}$ apresentou valor de $\mathrm{L}^{*}$ superior $(\mathrm{p}<0,05)$ à formulação $C$ e valores de $a^{*}$ e $b^{*}$ inferiores $(p<0,05)$ à formulação $C$ (tabela 3 ). Segundo Lindahl et al. ${ }^{(16)}$, os valores de vermelho $\left(\mathrm{a}^{*}\right)$ apresentam correlações altas com o conteúdo de pigmentos e pelas formas da mioglobina quase na mesma extensão. Ou seja, a redução dos valores de $a^{*}$ na formulação FMC provavelmente está relacionada à redução do teor relativo de mioglobina causado pelo menor percentual do conteúdo cárneo utilizado nessa formulação.

Pedroso et al. ${ }^{(12)}$ relataram que a adição de amido de mandioca (0 a $\left.2 \%\right)$ e carragena ( 0 a $\left.1 \%\right)$ em apresuntado de peru não afetou significativamente os valores de $\mathrm{L}^{*} \mathrm{e} \mathrm{a}^{*}$. Para os valores de $\mathrm{b}^{*}$, a carragena apresentou um efeito significativo sobre o produto. Teixeira et al. ${ }^{(17)}$, ao realizarem estudo com apresuntado com substituição parcial do conteúdo cárneo por farinha de yacon (0 a 5\%), relataram que as formulações com farinha de yacon apresentaram uma tonalidade mais escura e com uma maior participação de tonalidade amarela. 
Tabela 3. Características físico-químicos (média \pm erro padrão) de apresuntado elaborado com ou sem fibra de ervilha

\begin{tabular}{ccc}
\hline Características & $\mathbf{C}^{\mathbf{l}}$ & FMC $^{\mathbf{2}}$ \\
\hline $\mathrm{pH}$ & $5,86 \pm 0,01^{\mathrm{a}}$ & $5,88 \pm 0,01^{\mathrm{a}}$ \\
$\mathrm{L}^{*}$ & $55,64 \pm 0,16^{\mathrm{b}}$ & $57,06 \pm 0,01^{\mathrm{a}}$ \\
$\mathrm{a}^{*}$ & $5,41 \pm 0,11^{\mathrm{a}}$ & $4,45 \pm 0,03^{\mathrm{b}}$ \\
$\mathrm{b}^{*}$ & $5,28 \pm 0,05^{\mathrm{a}}$ & $4,98 \pm 0,04^{\mathrm{b}}$ \\
Perda por gotejamento & $0,97 \pm 0,04^{\mathrm{a}}$ & $0,85 \pm 0,03^{\mathrm{a}}$ \\
Dureza (g) & $3297 \pm 61^{\mathrm{a}}$ & $2320 \pm 79^{\mathrm{b}}$ \\
Elasticidade (mm) & $0,94 \pm 0,02^{\mathrm{a}}$ & $0,92 \pm 0,03^{\mathrm{a}}$ \\
Coesividade & $0,36 \pm 0,07^{\mathrm{a}}$ & $0,45 \pm 0,06^{\mathrm{a}}$ \\
Mastigabilidade (g.mm) & $1327 \pm 19^{\mathrm{a}}$ & $725 \pm 16^{\mathrm{b}}$ \\
\hline
\end{tabular}

${ }^{1}$ Controle: formulação similar a comercial; ${ }^{2} \mathrm{Fibra} / \mathrm{Menos}$ Carne: redução de $5 \%$ de carne, adição de $1 \%$ de fibra de ervilha e $4 \%$ de água.

Letras minúsculas diferentes em uma mesma linha indicam diferenças significativas $(\mathrm{p}<0.05)$ entre os tratamentos.

A porcentagem de perdas no teste de gotejamento não apresentou diferença $(p>0,05)$ entre as formulações (Tabela 3), demonstrando que a fibra de ervilha adicionada (1\%) foi capaz de incorporar a maior quantidade de água adicionada na formulação FMC (4\%). Pedroso et al. ${ }^{(12)}$ obtiveram 0,91 a $8,18 \%$ de perdas no resfriamento de presunto cozido de peru adicionado de amido ( 0 a $2 \%$ ) e carragena (0 a 1\%). Ao contrário do que foi encontrado no presente trabalho, os mesmos autores relatam menores perdas para as formulações que continham amido (2\%).

$\mathrm{Na}$ análise do perfil de textura, os parâmetros de elasticidade e coesividade não diferiram $(\mathrm{p}>0,05)$ entre os tratamentos. Em relação à dureza e mastigabilidade, a formulação FMC apresentou valores inferiores $(\mathrm{p}<0,05)$ ao $\mathrm{C}$, demonstrando que a substituição de parte da carne por fibra de ervilha e água no tratamento FMC gerou um produto mais macio. Ambos os parâmetros estão correlacionados, uma vez que a mastigabilidade é definida como o produto da dureza pela coesividade e elasticidade $^{(18)}$. Dessa forma, o valor inferior encontrado para a mastigabilidade na formulação FMC é decorrente da redução da dureza nessa formulação. Esses resultados corroboram com a afirmação de Aleson-Carbonell et al. ${ }^{(19)}$ de que uma diluição de insumos não cárneos em sistemas de proteína cárnea geralmente reduz o valor instrumental da textura do alimento preparado. Esses ingredientes tendem a reter maiores percentuais de água e gordura na matriz proteica, tornando o alimento mais macio. Teixeira et al. ${ }^{(17)}$, ao realizarem estudo com apresuntado com substituição parcial do conteúdo cárneo por farinha de yacon (0 a 5\%), encontraram maior índice de dureza, elasticidade e mastigabilidade nos tratamentos com maiores concentrações de farinha.

Os resultados da avaliação sensorial estão expressos na Tabela 4. Observa-se que as notas atribuídas pelos provadores para os atributos aroma, aparência, textura e sabor não apresentaram diferenças ( $p>0,05)$ entre as duas formulações. Já em relação à aceitabilidade geral a formulação FMC, o resultado apresentou média de notas superior $(\mathrm{p}<0,05)$ ao $\mathrm{C}$. Apesar dos atributos aroma, aparência, textura e sabor avaliados não terem apresentando diferenças, as maiores notas foram atribuídas para 
o tratamento fibra, sempre acima do "Gostei regularmente" (nota 7 da escala utilizada). Dessa forma, verificou-se que a redução do conteúdo cárneo e a aplicação de fibra de ervilha na formulação do apresuntado causaram efeito positivo na aceitação pelos consumidores.

Apesar da diferença $(\mathrm{p}<0,05)$ observada no parâmetro instrumental dureza durante a análise de perfil de textura, na avaliação sensorial, os provadores não detectaram diferença $(p>0,05)$ entre os tratamentos quanto ao atributo textura.

Sordi et al. ${ }^{(13)}$ avaliaram o grau de aceitação de presunto acrescido de 1,5\% de fibra solúvel de trigo e presunto adquirido comercialmente através de escala hedônica e não encontraram diferença significativa entre as amostras. Pedroso et al. ${ }^{(12)}$ avaliaram a aceitabilidade de presuntos com adição de amido de mandioca ( 0 a $2 \%$ ) e carragena ( 0 a $1 \%$ ) e também relataram que as amostras de presunto de peru não apresentaram diferenças significativas quanto à aceitabilidade.

Tabela 4. Aceitação sensorial (média \pm erro padrão) de apresuntado formulado com ou sem fibra de ervilha

\begin{tabular}{ccc}
\hline Atributos & $\mathbf{C}^{\mathbf{l}}$ & FMC $^{\mathbf{2}}$ \\
\hline Aroma & $6,85 \pm 0,20^{\mathrm{a}}$ & $7,15 \pm 0,18^{\mathrm{a}}$ \\
Aparência & $6,86 \pm 0,19^{\mathrm{a}}$ & $7,06 \pm 0,17^{\mathrm{a}}$ \\
Textura & $7,15 \pm 0,17^{\mathrm{a}}$ & $7,23 \pm 0,18^{\mathrm{a}}$ \\
Sabor & $7,15 \pm 0,19^{\mathrm{a}}$ & $7,41 \pm 0,17^{\mathrm{a}}$ \\
Aceitabilidade Geral & $7,05 \pm 0,16^{\mathrm{b}}$ & $7,48 \pm 0,15^{\mathrm{a}}$ \\
\hline${ }^{1}$ Controle: formulação similar a comercial; ${ }^{2} \mathrm{Fibra} /$ Menos Carne: redução de $5 \%$ de carne, adição de $1 \%$ de fibra \\
de ervilha e 4\% de água. \\
Letras minúsculas diferentes em uma mesma linha indicam diferenças significativas (p<0.05) entre os \\
tratamentos.
\end{tabular}

\section{Conclusão}

Nas condições avaliadas no presente trabalho, conclui-se que é possível utilizar a fibra de ervilha em apresuntado como substituto parcial de carne, com redução de custo e aumento da aceitação geral, sendo que esse tipo de fibra se mostrou eficiente no equilíbrio da formulação e não prejudicou a maioria das características físico-químicas do produto.

\section{Agradecimentos}

Agradeço a CAPES pela bolsa de mestrado e a empresa LABONATHUS BIOTECNOLOGIA INTERNACIONAL - São Paulo, Brasil pela doação da fibra de ervilha.

\section{Referências}

1. Junior ASM. Considerações importantes durante o processamento de carcaças suínas. Rev Nac Carne. 2004;328:82-5.

2. Corrêa I. O árduo caminho da Suinocultura Brasileira. Avic Suinocult Ind. 1990;80(970):8798.

3. Grigelmo-Miguel N, Abadias-Seros M, Martin Belloso O. Characterisation of low-fat high dietary fiber 
frankfurters. Meat Sci. 1999;52:247-56.

4. Rossell CM, Rojas JA, De Barber C. Influence of hydrocolloïds in dough rheology and bread quality. Food Hydrocoll. 2001;15:75-82.

5. Hur SJ, Lim BO, Park GB, Joo ST. Effect of various fiber additions on lipid digestion during in vitro digestion of beef patties. J Food Sci. 2009;74(9):C653-C657. Available from: http://onlinelibrary.wiley.com/doi/10.1111/j.1750-3841.2009.01344.x/pdf.

6. Kumar V, Biswas AK, Chatli MK, Sahoo J. Effect of banana and soybean hull flours on vacuum packaged chicken nuggets during refrigeration storage. Int J Food Sci Technol. 2011;46(1):122-29. Available from: http://onlinelibrary.wiley.com/doi/10.1111/j.1365-2621.2010.02461.x/pdf.

7. García ML, Dominguez R, Galvez MD, Casas C, Selgas MD. Utilization of cereal and fruit fibers in low fat dry fermented sausages. Meat Sci. 2002; 60:227-36.

8. Association of Official Analytical Chemistry. Official methods of analysis. 18nd ed. Gaithersburg: AOAC International; 2007. vol 1.

9. Bligh EG, Dyer WJ. A rapid method of total lipid extration and purification. Can J Biochem Physiol. 1959;37(8):911-17.

10. Brasil. Ministério da Agricultura, Pecuária e Abastecimento. Instrução Normativa n. 20 de 31/07/2000. Brasília: MAPA; Republicado em 03/08/2000. Available from: http://www.agricultura.gov.br.

11. Beserra FJ et al. Desenvolvimento e caracterização físico-química de embutido cozido tipo apresuntado de carne caprino. Cienc Rural. 2003;33(6):1141 -47. Available from: http://www.scielo.br/pdf/cr/v33n6/a22v33n6.pdf.

12. Pedroso RA, Demiate IM. Avaliação da influência de amido e carragena nas características físico-químicas e sensoriais de presunto cozido de peru. Cienc Tecnol Aliment. 2008;28(1):24-31. Available from: http://www.scielo.br/pdf/cta/v28n1/04.pdf.

13. Sordi MG, Menoncin S, Cichoski AJ, Zitikoski JL, Waszczynskyj N. Presunto cozido com fibras. Hig Aliment;2006; 20(145): 55-60.

14. Huber E. Desenvolvimento de produtos cárneos reestruturados de frango (hambúrguer e empanado) com adição de fibras vegetais como substitutos totais de gordura [tese]. Florianópolis: Universidade Federal de Santa Catarina, Centro Tecnológico; 2012. Available from: https://repositorio.ufsc.br/bitstream/handle/123456789/100599/308821.pdf?sequence=1.

15. Junior LCOS, Rizzatti R, Brungera A, Shiavini TJ, Campos LFM, Scaldo Neto JF, Rodrigues LB, Dicke EL, Santos LR. Desenvolvimento de hambúrguer de carne de ovinos de descarte enriquecido com farinha de aveia. Cienc anim bras. 2009 out [citado 12 mar 2014]; 10(4):1128-34. Available from: http://www.revistas.ufg.br/index.php/vet/article/view/3794/5928.

16. Lindahl G, Lundstrom K, Tornberg E. Contribution of pigment content, myoglobin forms and internal reflectance to the color of pork loin and ham from pure breed pigs. Meat Sci. 2001;59(2):141-51. Available from: http://www.sciencedirect.com/science/article/pii/S030917400100064X.

17. Teixeira JA. Elaboração de apresuntado formulado com farinha e extrato de yacon (Smallanthus sonchifollius) [dissertação]. Lavras - Universidade Federal de Lavras; 2011. Available from: http://repositorio.ufla.br/handle/1/1585

18. Ramos EM, Gomide LAM. Avaliação da qualidade de carnes: fundamentos e metodologias.

Viçosa: Editora UFV; 2007.

19. Aleson-Carbonell L et al. Characteristics of beef burger as influenced by various types of lemon albedo. 
Innov Food Sci Emerg Technol. 2005; 6(2): 247-55.

20. Larrea V, Pérez-Munuera I, Hernando I, Quiles A, Llorca E, Lluch MA. Microstructural changes in Teruel dry-cured ham during processing. Meat Sci. 2007;76:574-82. Available from: http://www.sciencedirect.com/science/article/pii/S0309174007000320.

21. Serio J, Muniz CR, Freitas CAS, Lima JR, Souza Neto JA. 2009. Avaliação microbiológica e microscópica de presuntos fatiados e refrigerados. Alim Nutr. 2009;20(1):135-39. Available from: http://200.145.71.150/seer/index.php/alimentos/article/viewArticle/956. 